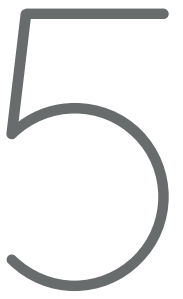

\title{
EL ÁMBITO DEL DISEÑADOR PROFESIONAL
}

Arq. Eduardo Jaramillo Porras 


\section{Introducción}

La Pontificia Universidad Católica del Ecuador y su extensión en Ibarra, de acuerdo a su plan de crecimiento académico y desarrollo institucional ha dado seguimiento a las tendencias y comportamiento de las actividades productivas y servicios de la región y del país con el objeto de identificar necesidades de capacitación y tecnificación de recursos humanos para alimentar a estos sectores.

El análisis del entorno socioeconómico y productivo de la región permitió a la Universidad identificar la necesidad de desarrollar un programa de formación profesional, orientado a capacitar recursos humanos para apoyar técnicamente las actividades industriales, pequeña industria y artesanía, para lo cual funda en 1982 la Escuela de Diseño con un plan curricular que se halla en vigencia y responde a las necesidades y características del perfil productivo del norte del país. Los objetivos que el nuevo programa académico propone son los siguientes:

Formar un Tecnólogo en un período de seis semestres con capacidad de trabajar intensa y extensamente en el desarrollo, concreción y materialización de las ideas y no sólo el enunciado de las mismas.

Formar un Profesional que conociendo su medio socio-cultural y entorno físico como determinante, haga del diseño un medio de solución a un problema humano y no un fin en sí mismo. 


\section{El Diseñador Profesional y su ámbito}

Para precisar el ámbito del diseñador profesional es necesario tener presente un conjunto de definiciones y dar respuesta a varias interrogantes:

a) Qué es el Diseño (funciones y objetivos).

b) Quién debe ser el Diseñador, su influencia y cuál su perfil profesional.

c) Dónde se forma.

d) Identificar su relación (Estado - Educación Superior - Sector Productivo).

e) Análisis de la demanda de diseñadores.

\section{Que es el Diseño}

Existe una variedad de conceptualizaciones sobre el diseño y su función: Cristopher Jones, define así al diseño: "iniciar un cambio en las cosas realizadas por el hombre".

Tomás Maldonado le define como "ACTIVIDAD PROYECTUAL, que consiste en determinar las propiedades formales de los objetos producidos". Según esta definición, proyectar significa; coordinar, integrar y articular todos aquellos factores y funciones que, de una manera o de otra, participan en el proceso productivo de la forma del producto. Factores relativos al uso, función y consumo individual o social y factores relativos a su producción técnico-económicos, constructivos, sistemáticos, productivos y distributivos, las funciones que definen el diseño son:

Función Humana -Relación hombre - usuario (cualitativas):

Cualidad precio: demanda económica social del usuario.

Cualidad formal: necesidad estética cultural.

Cualidad de eficiencia: requerimientos prácticos y fisiológicos del usuario.

Función Técnica -Relación de la planeación y el proceso: 
Técnica directa (inherente al objeto).

Técnica indirecta (relación entre el objeto y otros).

Función Productiva - Relación de las partes de que el objeto se compone y de éstas con el medio físico:

Planeación - desarrollo, financiamiento y mercado.

Manufactura - Tecnología de producción.

\section{El diseñador, su influencia y su perfil}

El diseñador debe ser un recurso humano con una formación académica, cuyo papel dentro de la sociedad consistirá en manejar y expresar los complejos procesos de generación de nuestra cultura material con la finalidad de provocar la máxima productividad y satisfacción. Debe ser el coordinador de aquellos aspectos que intervienen en el proceso de origen y constitución de la forma de los productos y no sólo CONSTRUCTOR, PROYECTISTA Y FORMALIZADOR.

El diseñador debe ser un profesional con una formación técnica y cultural, capaz de integrarse en un equipo multidisciplinario de profesionales y técnicos que intervienen en el proceso de formulación, producción y evaluación del producto, consciente de la magnitud y complejidad de las necesidades de la comunidad y de los problemas de la producción. Debe integrar dentro de las soluciones que plantea diversos factores en un todo coherente. Su aporte es conjugar varios factores: Funcionales, técnicos, económicos y culturales.

El diseñador debe contribuir a la consolidación de los criterios de autenticidad desde el punto de vista de los valores de la comunidad, y al desarrollo de los valores estéticos y materiales de su cultura. Es un profesional que racionaliza la producción de objetos y elementos artesanales e industriales, valoriza lo "nuestro" sin perder la proyección de las diferentes corrientes del diseño. Es un técnico que mejora, diversifica e incrementa en calidad y cantidad la producción y que para ello domina el conocimiento de su medio socio-cultural y entorno físico como determinantes que hacen del diseño un medio de solución a un problema y no un fin en sí mismo. 
El diseñador es un profesional que debe integrarse fácilmente a los centros de producción artesanal, pequeña industria e industria, donde el diseño es una necesidad, para lo cual debe garantizar una producción en variedad y calidad y satisfacer las demandas de nuevos prototipos que diversifiquen la producción.

\section{Formación Profesional y sector productivo}

Existe un desfase entre la capacitación formal en proyectar y diseñar que se imparte en las instituciones de enseñanza superior y las exigencias concretas del sector productivo.

Es evidente la necesidad de modificar la estructura y enfoque de las disciplinas productivas en las instituciones de enseñanza, una mayor vinculación con la realidad a través del enfoque teórico, que permita el acceso del trabajo proyectual a aquellos sectores y aspectos que en la actualidad se encuentran marginados debido a las condiciones impuestas por el Modelo de formulación y Diseño de productos vigente, transferido y adoptado en nuestro País.

Se necesita una mayor vinculación entre Estado - Universidad y Sector Productivo, para lo cual es necesario que los centros de Educación Superior y sus Escuelas Técnicas se constituyan en centros de cogestión tecnológica de apoyo permanente a las actividades de producción.

\section{Aporte de las instituciones de Educación Superior a la Industria y Artesanía}

Contribuyen al sector a través de las Escuelas de Diseño y la integración de profesionales altamente capacitados con una formación teórico-práctica.

Estimulan la creación de nuevos productos y sistemas que mejoren el nivel de vida de los habitantes del país. A través del establecimiento de relaciones de mutua cooperación con la Industria y Artesanía proyectando un sistema de apoyo en aspectos complementarlos, con miras a elevar el nivel académico y la calidad profesional de los educandos y ofrecer un apropiado servicio a los 
empresarios. La integración y promoción del elemento humano formado es un factor fundamental del progreso económico, tecnológico y cultural.

La Industria necesita del diseñador, formado en las escuelas de diseño; una industria en desarrollo necesita respuestas a las posibilidades y necesidades del país, no puede desenvolverse sin una dinámica propia en el desarrollo de sus productos y mejoramiento de las condiciones de producción.

La creatividad es el factor o cualidad más importante del ser humano que debe ser utilizada para aprovechar y optimizar el uso de los recursos no renovables y la tecnología. El diseño orienta el uso de la creatividad hacia el logro de una producción más eficiente y la consecución de productos más adecuados a las necesidades del mercado.

La integración de los profesionales en diseño a la industria representa la posibilidad de aprovechar óptimamente la tecnología desarrollando nuevos productos y más eficientes procesos, que permitan llegar a un uso más racional de los recursos humanos y naturales disponibles y aumentar el valor agregado de nuestras manufacturas. La opción de aumentar la competitividad de nuestros productos en los mercados internos y externos es una respuesta para el empresario a sus problemas y posibilidades técnicas y económicas con miras a un mayor desarrollo o a su permanencia en el mercado.

\section{Dependencia tecnológica}

Lo que es una actividad institucionalizada en países desarrollados no es más que una vaga realidad en los países periféricos o dependientes.

El carácter dependiente de nuestra tecnología y organización empresarial respecto a la industria extranjera ha implicado que conceptos como investigación, desarrollo de productos, incluso desarrollo industrial, correspondan más a buenas intenciones que a una realidad.

La mayor parte de nuestros productos son copia de otros elaborados con moldes y matrices de segunda o ya obsoletos en el exterior. 
La ampliación de los mercados internos y las tentativas de competir internacionalmente han mostrado el atraso tecnológico y de diseño. Las experiencias se fundan en excepciones a veces empíricas más que en una práctica regulada y sistematizada; tenemos una dependencia de las fuerzas productivas en nuestro país con el campo de los mercados de producción y en el de proposición de proyectos.

Sólo la acción integrada de los organismos relacionados en el diseño podrán provocar un cambio favorable para superar gradualmente esta dependencia. Sistema que se formula como propuesta en este documento.

\section{Análisis ocupacional}

El estudio más importante para determinar el ámbito del diseñador profesional es el del ANALISIS OCUPACIONAL.

El presente análisis se orienta a:

Identificar y clasificar las Unidades de Producción Industrial, servicios afines, relacionados con la demanda de diseñadores o Diseño Artesanal o Industrial.

Identificar y determinar el tamaño del mercado laboral actual y futuro para el diseñador.

Calcular la demanda actual y futura para este tipo de recurso.

Calcular la oferta actual y futura de diseñadores.

\section{Análisis de la demanda}

Para el análisis de la demanda de diseñadores (oferta de puestos de trabajo), se ha hecho una identificación y clasificación según el tipo de objeto producido o servicio prestado de todas las empresas industriales y de servicios que demanden o utilicen servicios profesionales de diseñadores con el objeto de caracterizar y determinar el universo de estudio en su tamaño y localización geográfica. 
a) Tamaño: Para determinar el tamaño del universo de estudio se tomó como unidad de análisis a todas las empresas de producción industrial y de servicios, agrupándoles según la especialización, sin discriminación de tamaño de la unidad, capital o volumen de producción.

b) Localización Geográfica: Todas las empresas industriales y de servicios que se localizan en las provincias del Carchi, Imbabura y Pichincha, por considerar geográficamente a estas provincias de influencia directa e inmediata en la determinación del mercado laboral actual, vinculadas con las actividades de la Universidad Católica Sede Ibarra.

Cuadro No. 1.- Empresas de producción industrial, según especialidad, número de crecimiento absoluto, calculados por períodos de cuatro años.

\begin{tabular}{lcccc}
\multicolumn{1}{c}{ TIPO DE EMPRESAS No. UPI } & No. UPI & No. UPI & No. UPI \\
& $\mathbf{1 9 8 2}$ & $\mathbf{1 9 8 6}$ & $\mathbf{1 9 9 0}$ & $\mathbf{1 9 9 4}$ \\
Maderas tratadas & 60 & 65 & 70 & 75 \\
Muebles de madera & 80 & 86 & 92 & 98 \\
Muebles de acero & 50 & 54 & 58 & 62 \\
Textiles & 36 & 39 & 42 & 45 \\
Cerámica & 20 & 22 & 24 & 26 \\
Artegráfico-Serigrafía & 85 & 91 & 98 & 105 \\
Plásticos y embalaje & 58 & 62 & 67 & 73 \\
Metálicos en general & 40 & 43 & 46 & 49 \\
Artesanal en general & 400 & 430 & 462 & 496 \\
Industria en general & 340 & 366 & 394 & 424 \\
& \multicolumn{4}{c}{} \\
TOTAL EMPRESAS & $\mathbf{1 . 1 6 9}$ & $\mathbf{1 . 2 5 8}$ & $\mathbf{1 . 3 5 3}$ & $\mathbf{1 . 4 5 3}$ \\
Fuente: Guía industrial y comercial del Ecuador, I.N.E.C., A.N.D.E. \\
Elaboración: CEDITUR - PUCE, Ibarra
\end{tabular}


Cuadro No. 2.- Empresas de producción de diseño (servicios) según especialidad, número y crecimiento absoluto, calculado por períodos de cuatro años.

$\begin{array}{lcccc}\text { TIPO DE EMPRESAS } & \mathbf{1 9 8 2} & \mathbf{1 9 8 6} & \mathbf{1 9 9 0} & \mathbf{1 9 9 4} \\ \text { Decoración } & 80 & 86 & 96 & 109 \\ \text { Creación, logotipo, planes } & 40 & 43 & 48 & 54 \\ \text { Diseño publicitario } & 86 & 92 & 103 & 116 \\ \text { Arquitectura } & 1.200 & 1.290 & 1.445 & 1.632 \\ \text { Diseño general } & 90 & 97 & 109 & 123 \\ & & & & \\ \text { TOTAL AMPRESAS } & \mathbf{1 . 4 9 6} & \mathbf{1 . 6 0 8} & \mathbf{1 . 8 0 1} & \mathbf{2 . 0 3 4}\end{array}$

Fuente y elaboración Idem

Cuadro No. 3.- Empresas de producción industrial y diseño según tipo, número y crecimiento absoluto calculado por períodos de cuatro años.

$\begin{array}{lllll}\text { TIPO DE EMPRESAS } & \mathbf{1 9 8 2} & \mathbf{1 9 8 6} & \mathbf{1 9 9 0} & \mathbf{1 9 9 4} \\ \text { Producción industrial UPI } & 1.169 & 1.258 & 1.353 & 1.453 \\ \text { Producción diseño UPD } & 1.496 & 1.608 & 1.801 & 2.034 \\ & & & & \\ \text { TOTAL EMPRESAS } & \mathbf{2 . 6 6 5} & \mathbf{2 . 8 8 6} & \mathbf{3 . 1 5 4} & \mathbf{3 . 4 8 7}\end{array}$

Fuente de elaboración: Idem

Del análisis del cuadro número 3 que totaliza el número de empresas de producción industrial y empresas de diseño (Servicios) localizadas en las provincias de Carchi, Imbabura y Pichincha se concluye que, a pesar de haber utilizado tasas de crecimiento UPI - $7.5 \%$ y (UPD - $7.5 \%$ y $12 \%$ ) muy conservadoras, es bastante significativo el número de unidades de producción que están relacionadas con la demanda permanente de este insumo industrial, convirtiéndose en un óptimo y selectivo mercado laboral para profesionales en diseño.

\section{Mercado laboral}

Este análisis está orientado a determinar cuantitativamente la oferta actual y futura de puestos de trabajo, para lo cual se tomó como unidad de análisis a grupos de 5 a 6 empresas de la misma especialidad, tanto en producción industrial (UPI) como de producción de diseño (UP) 
con el objeto de determinar una media específica ya que el comportamiento de la demanda no es igual.

Cuadro No. 4.- P. T/UPG y no absoluto de puestos de trabajo, según el tipo de empresa calculando en períodos de 4 años.

\section{No. DE PUESTOS DE TRABAJO}

Tipo de empresa

Producción industrial

Producción diseño
P.T/UPG

0.4475

0.370

TOTAL GENERAL $\mathbf{0 . 4 1 6}$
Fuente: Investigación de campo

Elaboración: CEDITUR - PUCE - Ibarra $\begin{array}{llll}1982 & 1986 & 1990 & 1994\end{array}$

$\begin{array}{llll}555 & 598 & 643 & 691\end{array}$

$553 \quad 596 \quad 666 \quad 762$

P.T/UPD: Media de relación entre P.T. y Unidad de Prod. Indus. y Diseño 0.416: Media de relación general para empresas que demandan diseñadores.

Del análisis del cuadro se desprende: que la media de relación general (P. T. UPG) es bastante significativa en tamaño (0.416) y que su tendencia en el futuro sería acrecer en la medida que las empresas de producción industrial y de diseño (servicios) se desarrollen y modernicen y que gradualmente disminuya la dependencia exterior en lo referente al consumo indiscriminado de diseños y prototipos, situación que define a que un volumen considerable de la producción industrial y artesanal ecuatoriana carezca, en su contenido, de valores estéticos y funcionales identificados con su cultura regional nacional. Determina también el cuadro un elevado número absoluto de puestos de trabajo.

Determina que el ámbito del diseñador profesional en el Norte del País es amplio y con responsabilidades, por lo que propongo que este estudio se generalice para el resto del País o se lo ponga al día si ya existe.

\section{Propuesta}

Formación de un CENTRO DE GESTIÓN CIENTÍFICA TECNOLÓGICA PARA EL DESARROLLO DEL DISEÑO, que regule y garantice el establecimiento de una apropiada relación 
PARTICIPA:

a) Sector Público (Estado).

b) Sector de Educación Superior (Universidades - Esc. Politécnicas).

c) Sector Productivo (Privado, Industria y Artesanía).

OBJETIVOS: Capacitación y formación de diseñadores en diferentes especializaciones.

a) Investigación e información tecnológica especializada.

b) Prestación de servicios técnicos de los tres sectores.

Se propone la creación de un Comité (ESTADO - EDUCACIÓN SUPERIOR - SECTOR PRODUCTIVO), que regule y garantice el establecimiento de una apropiada relación entre los tres sectores.

OBJETIVO: Establecer relaciones permanentes y de mutuo beneficio para los tres sectores mediante un sistema de cooperación directa que asegure la obtención de resultados positivos. Se divide en dos órganos principales:

a) EL OPERATIVO: Encargado de analizar, programar, ejecutar y evaluar las actividades que impulsen el desarrollo del programa.

b) COMITE ASESOR: Opera como órgano de consulta que orienta al Comité Operativo para la fijación y desarrollo de programas especiales reforzado por subcomités.

COMITE: ESTADO - UNIVERSIDAD - SECTOR PRODUCTIVO conformado por los representantes de la industria privada, gremios de la producción, entidades oficiales así como por las directivas de profesores y estudiantes de las universidades. 